\title{
FUNCTIONS SATISFYING ELEMENTARY RELATIONS
} BY

\author{
MICHAEL F. SINGER
}

\begin{abstract}
In this paper we deal with the following problems: When do the solutions of a collection of differential equations satisfy an elementary relation, that is, when is there an equation of the form $R=0$ where $R$ is some algebraic combination of logarithmic, exponential and algebraic functions involving solutions of our differential equations? If such relations exist, what can they look like? These problems are given an algebraic setting and general forms for such relations are exhibited. With these, we are able to show that certain classes of functions satisfy no elementary relations.
\end{abstract}

Introduction. The simplest case of determining when a solution of a differential equation satisfies an elementary relation is that of the single equation $y^{\prime}=\alpha$ where $\alpha$ is an elementary function, that is, a function expressible in terms of a finite number of algebraic operations, logarithms, and exponentials. We are then asking, when does the integral $y$ of $\alpha$ satisfy some elementary relation (for example, $\exp \left(y^{1 / 2}\right)+z^{2}+(\log (\log y))$. $(\exp z y)=0)$ ?

In [4], [5], Ritt answered this question by showing that if $y$ satisfies an elementary relation, it must then actually be elementary. This implies, for example, that no elliptic integral satisfies an elementary relation.

In Theorem 1, we generalize this result to consider the collection of equations $y_{1}^{\prime}=\alpha_{1}, \ldots, y_{n}^{\prime}=\alpha_{n}$ where the $\alpha_{i}$ are in some differential field $K$, and show that if the solutions $y_{1}, \ldots, y_{n}$ satisfy an elementary relation (such as $\left.y_{1}\left(\exp \left(\pi \log y_{2}\right)\right)+z^{2}\left(y_{3}^{2}-1\right)^{1 / 2}=0\right)$, then there is some nontrivial linear

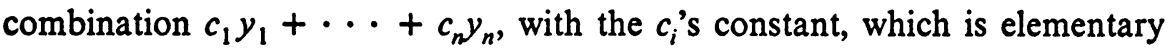
with respect to $K$. With this result, we are able to show that certain classes of integrals satisfy no elementary relations.

The next case to consider is that of a first order differential equation $F\left(z, y, y^{\prime}\right)=0$, where $F$ is a polynomial in $y$ and $y^{\prime}$ with coefficients in $\mathbf{C}(z)$. This case was originally treated by Mordukhai-Boltovski [4]. He showed that if a solution $y$ of such an equation satisfies an elementary relation, then it satisfies a relation of the form

$$
c_{1} \log \varphi_{1}(z, y)+\cdots+c_{n} \log \varphi_{n}(z, y)+\varphi_{0}(z, y)=0,
$$

Received by the editors November 27, 1974.

AMS (MOS) subject classifications (1970). Primary $12 \mathrm{HOS.}$ 
where the $\varphi_{i}$ 's are algebraic functions of $z$ and $y$ and the $c_{i}$ 's are constants. This result will be generalized in Theorem 2 to include differential equations of arbitrary order over arbitrary differential fields of characteristic zero.

This paper is divided into six sections. In $\S \S 1$ and 2 we will take care of some differential preliminaries leading up to a definition of elementary relations. $\$ 3$ contains a statement and proof of the Lemma which is the heart of this paper. $\$ \S 4$ and 5 contain precise statements and proofs of Theorems 1 and 2 . $\$ 6$ contains comments about further generalizations of these results.

1. Generalized elementary extensions. Throughout this paper, all fields will be of characteristic zero, $\mathbf{Z}$ will denote the integers, $\mathbf{Q}$ will denote the rationals, and $\mathbf{C}$ will denote the complex numbers.

If $E$ is a field, a map $D: E \rightarrow E$ is called a derivation if $D(a+b)=D a+$ $D b$ and $D(a b)=(D a) b+a(D b)$ for all $a, b$ in $E$. A differential field is a field $E$ with a set of derivations $\mathscr{D}=\{D\}$. (Note: we do not assume that the derivations commute.) A subfield $k$ of $K$ which is closed under the derivations $D$ of $K$ is called a differential subfield. If $c$ is in $K$ and $D c=0$ for all $D$ in $\mathscr{D}$, we say $c$ is a constant of $K$. The set of constants can be seen to form a differential subfield of $K$. If $k$ is a differential subfield of $K$ and $u \in K$ then $k\langle u\rangle$ denotes the smallest differential subfield of $K$ containing $k$ and $u$.

Let $E$ be a differential field with derivations $\mathscr{D}=\{D\}$, and $k$ a differential subfield. An element $\theta$ in $E$ is said to be an elementary integral with respect to $k$ if there exist constants of $k, d_{1}, \ldots, d_{n}$, and elements $v, u_{1}, \ldots, u_{n}$ in $k$ such that

$$
D \theta=\sum_{i=1}^{n} d_{i} \frac{D u_{i}}{u_{i}}+D v \text { for all } D \text { in } \mathscr{D} \text {. }
$$

A nonzero element $\theta$ in $E$ is said to be an exponential of an elementary integral with respect to $k$ if there exist constants of $k, c_{1}, \ldots, c_{n}$, and elements $v$, $u_{1}, \ldots, u_{n}$ in $k$ such that

$$
\frac{D \theta}{\theta}=\sum_{i=1}^{n} c_{i} \frac{D u_{i}}{u_{i}}+D v \text { for all } D \text { in } \mathscr{D}
$$

A differential field $K$ is said to be a generalized elementary extension of a differential subfield $k$ if: (1) $k$ and $K$ have the same field of constants, and (2) there exist differential intermediate fields $k=K_{0} \subset \cdots \subset K_{n}=K$ such that $K_{i}=K_{i-1}\left(\theta_{i}\right)$ where each $\theta_{i}$ is an elementary integral or an exponential of an elementary integral or algebraic with respect to $K_{i-1}$.

As usual, an element $u$ in $E$ is said to be a logarithm (exponential) over $k$ if

$$
D u=\frac{D v}{v} \quad\left(\frac{D u}{u}=D v\right) \text { for some } v \text { in } k \text { and all } D \text { in } \mathscr{D} \text {. }
$$


A differential field $K$ is said to be an elementary extension of a differential subfield $k$ if: (1) $K$ and $k$ have the same field of constants, and (2) there exist differential subfields $k=K_{0} \subset \cdots \subset K_{n} \subset K$ such that $K_{i}=K_{i-1}\left(\theta_{i}\right)$ where $\theta_{i}$ is a logarithm, exponential or algebraic with respect to $K_{i-1}$. An element $w$ of some big differential extension of $k$ is said to be elementary with respect to $k$ if it lies in an elementary extension of $k$. Note that we have required elementary and generalized elementary extensions to have the same constant field as the base field. This convention will hold throughout the paper.

After all these definitions, some remarks are in order. First of all, a logarithm is an elementary integral and an exponential is an exponential of an elementary integral. Therefore, an elementary extension is a generalized elementary extension.

Secondly, any generalized elementary extension of $k$ lies in an elementary extension of $k$. The main tool in showing this will be the following proposition whose statement and proof are very similar to Lemma 3.9 in [3], but included here for convenience.

Proposition 1. Let $K$ be a differential field of characteristic 0 with derivations $\Phi$. Let $\zeta$ be an element of a larger differential field with $D \zeta$ in $K$ for all $D$ in $\mathscr{D}$. Assume there is no $\xi$ in $K$ such that $D \xi=D \zeta$ for all $D$ in $\mathscr{D}$. Then $\zeta$ is transcendental over $K$ and $K\langle\zeta\rangle$ has the same field of constants as $K$.

Proof. Assume $\zeta$ is algebraic over $K$ and that $X^{n}+a_{n-1} X^{n-1}+\cdots+$ $a_{0}$ is the minimal polynomial of $\zeta$. Since $\zeta^{n}+a_{n-1} \zeta^{n-1}+\cdots+a_{0}=0$, we can apply any $D$ in $\mathscr{D}$ and get

$$
n(D \zeta) \zeta^{n-1}+\left(D a_{n-1}\right) \zeta^{n-1}+\cdots=0
$$

so $n D \zeta=-D a_{n-1}$ i.e., $-a_{n-1} / n$ would be an element of $K$ such that $D\left(-a_{n-1} / n\right)=D \zeta$ for all $D$ in $\mathscr{D}$. This would be a contradiction.

Next I will show that $K\langle\zeta\rangle$ has no new constants. Assume an element of the form $b_{n} \zeta^{n}+b_{n-1} \zeta^{n-1}+\cdots+b_{0}$ is a constant. For every $D$ in $\mathscr{D}$,

$$
\begin{aligned}
0 & =D\left(b_{n} \zeta^{n}+b_{n-1} \zeta^{n-1}+\cdots+b_{0}\right) \\
& =\left(D b_{n}\right) \zeta^{n}+\left(n b_{n}(D \zeta)+\left(D b_{n-1}\right)\right) \zeta^{n-1}+\ldots
\end{aligned}
$$

So we have $D b_{n}=n b_{n}(D \zeta)+D b_{n-1}=0$. Therefore,

$$
D \zeta=D\left(-\left(b_{n-1} / n b_{n}\right)\right) \text { for all } D \text { in } \mathscr{D}
$$

which is a contradiction unless $n=0$, i.e., unless our element was in $K$ to start with. Now assume we have a constant of the form $f / g$, where $f$ and $g$ are polynomials in $\zeta$ in lowest terms and $g$ is monic of degree bigger than or equal to one. Then for any $D$ in $\mathscr{D}$, 


$$
D(f / g)=(D f g-f D g) / g^{2}=0
$$

so $f / g=D f / D g$ contradicting the fact that $f / g$ is in lowest terms. This completes the proof of the proposition.

This proposition can be used in the following way. Let $K$ be a differential field and, for each $D$ in $\mathscr{D}$, let $\alpha_{D}$ be an element in $K$. Assume that there is no $\xi$ in $K$ such that $D \xi=\alpha_{D}$ for each $D$ in $\mathscr{D}$. Let $K(\zeta)$ be a simple transcendental extension of $K$ and extend each $D$ in $\mathscr{D}$ to a derivation of $K(\zeta)$ by setting $D(\zeta)=\alpha_{D}$. Proposition 1 then assures us that $K(\zeta)=K\langle\zeta\rangle$ has the same field of constants as $K$.

Instead of rigorously proving that each generalized elementary extension lies in an elementary extension, I will work out one case, and then indicate how one is to proceed. Hopefully, what is lost in rigor is gained in comprehensibility and clarity of notation.

Let us assume that $K\left(\eta_{1}, \eta_{2}, \eta_{3}\right)$ is a generalized elementary extension of $K$ where $\eta_{1}$ is algebraic over $K$,

$$
D \eta_{2}=\sum_{i=1}^{l} c_{i} \frac{D U_{i, 2}}{U_{i, 2}}+D V_{2} \text { for all } D \text { in } \Phi
$$

with $U_{i, 2}$ and $V$ in $K\left(\eta_{1}\right)$ for $1 \leqslant i \leqslant l$ and

$$
\frac{D \eta_{3}}{\eta_{3}}=\sum_{i=1}^{k} d_{i} \frac{D U_{i, 3}}{U_{i, 3}}+D V_{3} \text { for all } D \text { in } \mathcal{D}
$$

with $U_{i, 3}$ and $V_{3}$ in $K\left(\eta_{1}, \eta_{2}\right), 1<i \leqslant k$. Now consider the field $K\left(\eta_{1}, \eta_{2}, \eta_{3}\right)$. Either this field contains an element $U_{1,2}^{*}$ such that

$$
D U_{1,2}^{*}=D U_{1,2} / U_{1,2} \text { for all } D \text { in } \mathcal{D}
$$

or, as noted immediately following Proposition 1, we can adjoin such an element without extending the field of constants. Similarly, we can adjoin, if necessary, an element $U_{2,2}^{*}$ such that

$$
D U_{2,2}^{*}=D U_{2,2} / U_{2,2}
$$

without extending the constants. Continuing in this way, we get a field $K\left(\eta_{1}, U_{1,2}^{*}, \ldots, U_{l, 2}^{*}\right)$ which is an elementary extension of $K$. Since the field $K\left(\eta_{1}, \eta_{2}, \eta_{3}, U_{1,2}^{*}, \ldots, U_{l, 2}^{*}\right)$ was constructed to contain no new constants, the elements $\eta_{2}$ and $\Sigma_{i=1}^{l} c_{i} U_{i, 2}^{*}+V$ differ by a constant in $K$. Thus $\eta_{2}$ is in $K\left(\eta_{1}, U_{1,2}^{*}, \ldots, U_{2, l}^{*}\right)$. Now, using Proposition 1 again, we can add, where necessary, elements $U_{i, 3}^{*}$ to $K\left(\eta_{1}, \eta_{2}, \eta_{3}, U_{1,2}^{*}, \ldots, U_{l, 2}^{*}\right)$ without enlarging the constants, such that

$$
D U_{i, 3}^{*}=D U_{i, 3} / U_{i, 3} \text { for } 1 \leqslant i<k \text { and all } D \text { in } \mathscr{D} \text {. }
$$

$K\left(\eta_{1}, U_{1,2}^{*}, \ldots, U_{l, 2}^{*}, U_{1,3}^{*}, \ldots, U_{k, 3}^{*}, \eta_{3}\right)$ is an elementary extension of $K$, and as we have seen, $K\left(\eta_{1}, \eta_{2}, \eta_{3}\right)$ is a subfield. In general, given a generalized 
elementary extension of $K$, we would throw in enough logarithms to make it into an elementary extension.

In particular, we can conclude that an elementary integral is elementary. Conversely, Liouville's theorem [6], says that if $K$ is a differential field with a single derivation ', and $y$ is a member of some elementary extension of $K$ with $y^{\prime}$ in $K$, then $y^{\prime}=v_{0}^{\prime}+\sum_{i=1}^{n} c_{i} v_{i}^{\prime} / v_{i}$ for some elements $v_{i}$ in $k$ and constants $c_{i}$. Another way of saying this is: if an integral $y$ of an element of $K$ is elementary, then $y$ is an elementary integral (this, of course, was why elementary integrals were so named). Since generalized elementary extensions lie in elementary extensions, why did we introduce them? The answer is that these extensions make the induction machinery in the proof of Lemma 1 work. If one considers the ordinary differential field $k=\mathbf{C}(z)$ with $z^{\prime}=1$ and $u=\pi \log z+\log (z+1)$, then $u$ is clearly elementary over $\mathbf{C}(z)$. One can show (see below) that any elementary tower that contains $u$ has at least transcendence degree 2 over $\mathbf{C}(z)$. Using generalized elementary towers, we can get away with a tower of transcendence degree 1 over $\mathbf{C}(z)$, since $u$ is an elementary integral. The proof of Lemma 1 proceeds by an induction on transcendence degree and involves constructing new towers. The economy offered by the notion of generalized elementary towers allows the induction to go through. Hopefully, this will be clearer after one has read the proof.

Proposition 2. If $K_{0}=\mathrm{C}(z) \subseteq K_{1} \subseteq \cdots \subseteq K_{n}$ is an elementary tower and $u$ is an element of $K_{n}$ such that $u^{\prime}=\pi / z+1 /(z+1)$, then the transcendence degree of $K_{n}$ over $K_{0}$ is bigger than or equal to 2.

Proof. Assume not.

Case 1. Transcendence degree $K_{n} / K_{0}=0$. In this case $u$ would be algebraic over $\mathbf{C}(z)$, and so (trace $u)^{\prime}=n(\pi / z+1 /(z+1)$ ) for some $n$ in $\mathbf{Z}$. This means that there would be a $v$ in $\mathrm{C}(z)$ such that $v^{\prime}=\pi / z+1 /(z+1)$. This is impossible since if we take the partial fraction decomposition for $v$ and differentiate term by term, we could not get any terms of the form $1 /(z+1)$ or $\pi / z$.

Case 2. Transcendence degree $K_{n} / K_{0}=1$. Here we will use some facts concerning function fields of one variable, for which the reader is referred to [2]. In this case, we can assume that there is a finite extension $k$ of $\mathbf{C}(z)$ and an element $t$ in $K_{n}$, transcendental over $k$ such that (i) $t^{\prime}=v^{\prime} / v$ or (ii) $t^{\prime} / t=v^{\prime}$ for some $v$ in $k$ and such that $u$ and $t$ are algebraically dependent over $k$. If (i) holds, we can apply the Kolchin-Ostrowski Theorem [1], which states: Let $F$ be a differential field with derivations $\{D\}$ and let $\eta_{1}, \ldots, \eta_{n}$, $\zeta_{1}, \ldots, \zeta_{m}$ be elements of an extension of $F$, having the same field of constants, such that $D \eta_{i} \in F, 1<i<n$, and $D \zeta_{i} / \zeta_{i} \in F, 1 \leqslant i \leqslant n$, for each $D$. If $\eta_{1}, \ldots, \eta_{n}, \zeta_{1}, \ldots, \zeta_{m}$ are algebraically dependent over $F$, then 
there exists a nontrivial linear combination $\Sigma c_{i} \eta_{i}$ of the $\eta_{i}$ 's with the $c_{i}$ 's constant, which lies in $F$ or $\exists \nu_{1}, \ldots, \nu_{m}$ in $\mathbf{Z}$, not all zero, s.t. $\Pi \zeta_{i}^{\nu_{1}} \in F$.

Using this we see that there exist constants $c_{1}$ and $c_{2}$ such that $c_{1} t+c_{2} u=$ $w$ is in $k$. This means that

$$
c_{1} \frac{v^{\prime}}{v} d z+c_{2} \frac{\pi}{z} d z+c_{2} \frac{1}{z+1} d z=d w .
$$

Note that the residue of $v^{\prime} / v d z$ at any place of $K_{n}$ is an integer corresponding to the order of $v$ at that place, and that the residue of $d w$ is always zero. If $c_{2}=0$, then $v$ would be in $\mathrm{C}$ and so would $t$, so we can assume $c_{2} \neq 0$. Let $P_{1}$ be a place lying above $(z)_{0}$ with ramification index $p_{1}$ and $P_{2}$ a place lying above $(z+1)_{0}$ with ramification index $p_{2}$. Taking residues at $P_{1}$ and $P_{2}$ we see that there are integers $n$ and $m$ such that $n c_{1}+p_{1} c_{2} \pi=0$ and $m c_{1}+p_{2} c_{2}$ $=0$. This would mean that $\pi$ is rational, a contradiction. If (ii) holds, then by the Kolchin-Ostrowski Theorem, we get that either $u$ is in $k$ or $t^{m}$, for some $m$, is in $k$. If the former holds, we are reduced to Case 1. If the latter holds, we get a contradiction since $t$ was assumed transcentental over $k$. This finishes the proof of Proposition 2.

For further reference, we note here that if $k$ is $\mathbf{C}\left(z_{1}, \ldots, z_{n}\right)$ with derivations $\partial / \partial z_{1}, \ldots, \partial / \partial z_{n}$, then an element of a generalized elementary extension of $k$ is called an elementary function of $n$ variables.

2. Elementary relations. Given an elementary function of $\boldsymbol{n}$ variables, we want to be able to substitute certain functions for the variables. In this section, I will give an algebraic formulation of this notion.

Let $k \subset K$ be differential fields with derivations $\mathscr{D}=\{D\}$ and $L$ a differential field with derivations $\Delta=\{\delta\}$. Assume that $k$ and $L$ have the same field of constants denoted by $\mathcal{C}$. Let $\alpha$ be an element of $K$, which is algebraic or an elementary integral or an exponential of an elementary integral with respect to $k$, and $\varphi$ an (algebraic) place from $K$ to $L$ with valuation ring $\theta$, such that $\varphi$ restricted to $C$ is the identity map. We say $\varphi$ is defined on $\alpha$ with respect to $k$ if $\alpha$ is in $\theta$ and either (a) $\alpha$ is algebraic over $k$ or (b) there exist elements $U_{1}, \ldots, U_{n}, V$ in $k \cap \theta$ with $\varphi\left(U_{i}\right) \neq 0$ for $1<i<$ $n$ and constants $c_{1}, \ldots, c_{n}$ in $C$ such that

$$
D \alpha=\sum_{j=1}^{n} c_{j} \frac{D U_{j}}{U_{j}}+D V \text { for each } D \text { in } \mathscr{D}
$$

and

$$
\delta(\varphi(\alpha))=\sum_{j=1}^{n} c_{j} \frac{\delta\left(\varphi\left(U_{j}\right)\right)}{\varphi\left(U_{j}\right)}+\delta(\varphi(V)) \text { for all } \delta \text { in } \Delta,
$$

or (c) $\varphi(\alpha) \neq 0$ and there exist elements $S_{1}, \ldots, S_{n}, T$ in $K \cap \theta$ with $\varphi\left(S_{i}\right) \neq 0$ for $1 \leqslant i \leqslant n$ and constants $d_{1}, \ldots, d_{n}$ in $\mathcal{C}$ such that 


$$
\frac{D \alpha}{\alpha}=\sum_{j=1}^{n} d_{j} \frac{D S_{j}}{S_{j}}+D T \text { for each } D \text { in } \mathscr{D}
$$

and

$$
\frac{\delta(\varphi(\alpha))}{\varphi(\alpha)}=\sum_{j=1}^{n} d_{j} \frac{\delta\left(\varphi\left(S_{j}\right)\right)}{\varphi\left(S_{j}\right)}+\delta(\varphi(T)) \text { for each } \delta \text { in } \Delta .
$$

Intuitively, this means that $\varphi$ preserves the property of being an elementary integral or exponential of an elementary integral.

Let $K_{0}$ and $L_{0}$ be differential fields with sets of derivatives $\mathscr{D}=\{D\}$ and $\Delta=\{\delta\}$. Assume that the field of constants of $K_{0}$ is the same as the field of constants of $L_{0}$ and call this field $\circlearrowright$. Let $\varphi_{0}$ be a place of $K_{0}$ onto $L_{0}$ which is the identity map on $\mathcal{C}$. Let $K_{n} \supset K_{n-1} \supset \cdots \supset K_{0}$ be a generalized elementary tower over $K_{0}$, where $K_{i}=K_{i-1}\left(\eta_{i}\right)$ and $L_{n} \supset L_{n-1} \supset \cdots \supset L_{0}$ be a generalized elementary tower over $L_{0}$ where $L_{i}=L_{i-1}\left(\theta_{i}\right)$. Let $\varphi$ be a place of $K_{n}$ onto $L_{n}$ with valuation ring $\theta$ and $\mathcal{E}$ some subset of $\mathscr{D}$. We say that $\left(K_{n}\right.$, $\left.L_{n}, \varphi\right)$ defines an elementary relation for $\left(K_{0}, L_{0}, \varphi_{0}, \mathcal{E}\right)$ if:

(1) $\varphi$ restricted to $K_{0} \cap \theta$ is $\varphi_{0}$.

(2) For each $i, 1 \leqslant i \leqslant n, \varphi$ is defined on $\eta_{i}$ with respect to $K_{i-1}$ and $\varphi\left(\eta_{i}\right)=\theta_{i}$.

(3) If $\alpha$ is some element of $\theta$ such that $\varphi(\alpha)=0$ and $D(\alpha)=0$ for all $D$ in $\delta$, then $\alpha=0$.

(4) There exists an $R$ in $\theta, R \neq 0$, such that $\varphi(R)=0$.

Let us see what these conditions intuitively stand for. Let $K_{0}=$ $\mathbf{C}\left(z_{1}, \ldots, z_{n}\right)$ with derivations $\partial / \partial z_{1}, \ldots, \partial / \partial z_{n}$ and $\mathcal{E}=$ $\left\{\partial / \partial z_{2}, \ldots, \partial / \partial z_{n}\right\}$. Let $\varphi_{0}$ represent substituting functions $f_{1}, \ldots, f_{n-1}$ for the variables $z_{2}, \ldots, z_{n}$ in $K_{0}$. Then $\varphi$ represents substituting $f_{1}, \ldots, f_{n-1}$ for $z_{2}, \ldots, z_{n}$ in some collection of elementary functions of $n$ variables. Condition 2 says that the differential structure of each of the generating functions $\eta_{i}$ is preserved. Condition 3 says that if a function $\alpha$ is independent of $z_{2}, \ldots, z_{n}$ and $\alpha\left(z_{1}, f_{1}, \ldots, f_{n-1}\right)=0$, then $\alpha=0$, which is clear since the substitution could have no effect on $\alpha$. Condition 4 says that there is some elementary function $R\left(z_{1}, \ldots, z_{n}\right)$, not identically zero, such that $R\left(z_{1}, f_{1}, \ldots, f_{n-1}\right)=0$, that is, $f_{1}, \ldots, f_{n-1}$ satisfy an elementary relation.

Let us look at some formal examples:

(a) Let $E$ be an ordinary differential field with derivation ', and $K_{0}=$ $E\left(W_{1}, \ldots, W_{n}\right)$ with $W_{1}, \ldots, W_{n} n$ algebraically independent elements. We can define derivations $\mathscr{D}=\left\{D_{0}, D_{1}, \ldots, D_{n}\right\}$ on $K_{0}$ where $D_{0}$ restricted to $E$ is ', $D_{0}\left(W_{i}\right)=0$ for $1<i \leqslant n, D_{i}(E)=0$ for $1 \leqslant i \leqslant n$, and $D_{i}\left(W_{j}\right)=1$ if $i=j$ and equals 0 otherwise. Let $L_{0}=E\left(w_{1}, \ldots, w_{n}\right)$ be an ordinary differential extension of $E$ with the same field of constants as $E$ and s.t. $w_{i}^{\prime} \in E$ 
for $1<i<n$. Let $\varphi_{0}$ be a place of $K_{0}$ to $L_{0}$ such that $\varphi_{0}\left(W_{i}\right)=w_{i}$ and $\varphi_{0}$ restricted to $E$ is the identity map and let $\mathcal{E}=\left\{D_{1}, \ldots, D_{n}\right\}$. We say that the integrals $w_{1}, \ldots, w_{n}$ satisfy an elementary relation if there exists $\left(K_{n}, L_{n}, \varphi\right)$ which defines an elementary relation for $\left(K_{0}, L_{0}, \varphi, \mathcal{E}\right)$.

(b) Let $y$ be a function (in a suitable domain) such that $y-\exp \left(y z^{-1}\right)=0$. We then see that $y^{\prime}=y^{2}\left(z y-z^{2}\right)^{-1}$. One can show that $y$ cannot be algebraic over $\mathbf{C}(z)$. Let $L_{0}=\mathbf{C}(z, y)$ be an ordinary differential field with a derivation $\delta$ such that $\delta z=1$ and $\delta y=y^{2}\left(z y-z^{2}\right)^{-1}$. Let $K_{0}=\mathrm{C}(z, Y)$ be a differential field with derivations $\mathscr{D}=\left\{D_{0}, D_{1}\right\}$ where $D_{0}(C)=0, D_{0}(z)=1$, $D_{0}(Y)=0$ and $D_{1}(C)=0, D_{1}(z)=0, D_{1}(Y)=1$. Let $\varphi_{0}$ be an isomorphism of $K_{0}$ and $L_{0}$ sending $z$ to $z$ and $Y$ to $y$. Let $K_{1}=K_{0}\left(\exp \left(Y z^{-1}\right)\right)$ and $L_{1}=L_{0}\left(\exp \left(y z^{-1}\right)\right)$ and $\varphi$ a place extending $\varphi_{0}$ such that $\varphi\left(\exp \left(Y z^{-1}\right)\right)=$ $\exp \left(y z^{-1}\right)$. Finally, let $R=Y-\exp \left(Y z^{-1}\right)$ and $\mathcal{E}=\left\{D_{1}\right\}$, then we can conclude that $\left(K_{1}, L_{1}, \varphi\right)$ defines an elementary relation for $\left(K_{0}, L_{0}, \varphi_{0}, \mathcal{E}\right)$.

There is one technical fact about elementary relations which I will need in the proof of Lemma 1. If there exists a triple $\left(K_{n}, L_{n}, \varphi\right)$ which defines an elementary relation for some $\left(K_{0}, L_{0}, \varphi_{0}, \delta\right)$ then there exists such a triple where the $\eta_{i}$ 's used in building $K_{n}$ satisfy the following property: if $\eta_{i}$ is transcendental over $K_{i-1}$ then there exist elements $v, v_{1}, \ldots, v_{n}$ in $\theta \cap K_{r-1}$ with $\varphi\left(v_{k}\right) \neq 0$ for $1<k<r$ and constants $c_{1}, \ldots, c_{r}$ such that either

(a)

$$
D \eta_{i}=\sum_{k=1}^{r} c_{k} \frac{D v_{k}}{v_{k}}+D v \text { for all } D \text { in } \mathscr{D} \text {, }
$$

$$
\delta \varphi\left(\eta_{i}\right)=\sum_{k=1}^{r} c_{k} \frac{\delta\left(\varphi\left(v_{k}\right)\right)}{\varphi\left(v_{k}\right)}+\delta(\varphi(v)) \text { for all } \delta \text { in } \Delta,
$$

and $c_{1}, \ldots, c_{r}$ are linearly independent over $\mathbf{Q}$; or

$$
\begin{aligned}
\frac{D \eta_{i}}{\eta_{i}} & =\sum_{k=1}^{r} c_{k} \frac{D v_{k}}{v_{k}}+D v \text { for all } D \text { in } \Phi, \\
\frac{\delta\left(\varphi\left(\eta_{i}\right)\right)}{\varphi\left(\eta_{i}\right)} & =\sum_{k=1}^{r} c_{k} \frac{\delta\left(\varphi\left(v_{k}\right)\right)}{\varphi\left(v_{k}\right)}+\delta(\varphi(v)) \text { for all } \delta \text { in } \Delta,
\end{aligned}
$$

and $1, c_{1}, \ldots, c_{r}$ are linearly independent over $\mathbf{Q}$.

We will start with a triple $\left(K_{n}, L_{n}, \varphi, \mathcal{E}\right)$ and show how at each stage we can fix the tower so that it satisfies the above property. Assume that for some $i$, with $\eta_{i}$ transcendental over $K_{i-1}$, there exist $u_{1}, \ldots, u_{n}, v$ in $\mathcal{O} \cap K_{i-1}$ with $\varphi\left(u_{k}\right) \neq 0$ for each $k$, and $c_{1}, \ldots, c_{n}$ constants, linearly dependent over $\mathbf{Q}$ such that

$$
D \eta_{i}=\sum_{k=1}^{n} c_{k} \frac{D u_{k}}{u_{k}}+D v \text { for all } D \text { in } \mathscr{D}
$$

and 


$$
\delta\left(\varphi\left(\eta_{i}\right)\right)=\sum_{k=1}^{n} c_{k} \frac{\delta\left(\varphi\left(u_{k}\right)\right)}{\varphi\left(u_{k}\right)}+\delta \varphi(v) \text { for all } \delta \text { in } \Delta .
$$

Suppose $c_{1}, \ldots, c_{r}$ form a basis for the $\mathbf{Q}$ span of $c_{1}, \ldots, c_{n}$. Say

$$
c_{r+j}=\sum_{i=1}^{r} \frac{\nu_{i j}}{\nu_{0}} c_{i} \text { for } 1 \leqslant j \leqslant n-r
$$

with each $\nu_{0}, \nu_{i j}$ in $\mathrm{Z}$. We get

$$
D \eta_{i}=\sum_{k=1}^{r} \frac{c_{k}}{\nu_{0}} \frac{D\left(u_{k}^{v} u_{r+1}^{v_{k, 1}} \cdots u_{n}^{\nu_{k, n-r}}\right)}{u_{k}^{v} u_{r+1}^{v_{k, 1}} \cdots u_{n}^{\nu_{k, n-r}}}+D v
$$

for all $D$ in $\mathscr{D}$. Setting $v_{k}=u_{k}^{\nu} u_{r+1}^{\nu_{k, 1}} \cdots u_{n}^{\nu_{k, n-r}}$, we get

$$
D \eta_{i}=\sum_{k=1}^{r} \frac{c_{k}}{\nu_{0}} \frac{D v_{k}}{v_{k}}+D v \text { for all } D \text { in } \mathscr{D} \text {. }
$$

Then we can see that $v_{k}$ is in $\theta \cap K_{i-1}$ and $\varphi\left(v_{k}\right) \neq 0$ for each $k$. The other properties of (a) can also be easily checked.

Now suppose that for some $i$, with $\eta_{i}$ transcendental over $K_{i-1}$, there exist $u_{1}, \ldots, u_{n}, u$ in $\theta \cap K_{i-1}$ and $c_{1}, \ldots, c_{n}$, constants, with $1, c_{1}, \ldots, c_{n}$ linearly dependent over $\mathbf{Q}$, such that

$$
D \eta_{i}=\sum_{k=1}^{n} c_{k} \frac{D u_{k}}{u_{k}}+D u \text { for all } D \text { in } \mathscr{Q} \text {. }
$$

Suppose $1, c_{1}, \ldots, c_{r}$ form a basis for the $\mathbf{Q}$ span of $c_{1}, \ldots, c_{n}$. Say

$$
c_{r+j}=\frac{\nu_{0 j}}{\nu_{0}}+\sum_{i=1}^{r} \frac{\nu_{i j}}{\nu_{0}} c_{i} \text { for } 1 \leqslant j \leqslant n-r
$$

with each $\nu_{0}, \nu_{i j}$ in $\mathbf{Z}$. We get

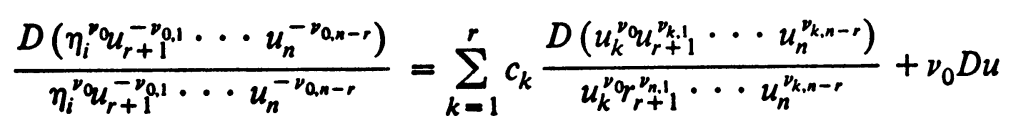

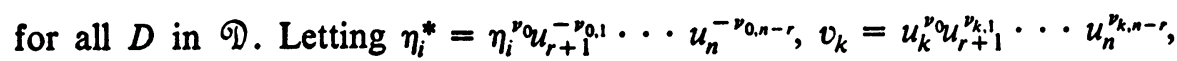
$v=v_{0} u$ we get

$$
\frac{D \eta_{i}^{*}}{\eta_{i}^{*}}=\sum_{k=1}^{r} c_{k} \frac{D v_{k}}{v_{k}}+D v \text { for all } D \text { in } \mathscr{D} \text {. }
$$

Again we can see that $v_{k}$ is in $\theta \cap K_{i-1}, \varphi\left(v_{k}\right) \neq 0$ for each $k, \eta_{i}^{*}$ is transcendental over $K_{i-1}$ and in the valuation ring of $\varphi$. Furthermore, $\eta_{i}$ is algebraic over $K_{i-1}\left(\eta_{i}^{*}\right)$. So we can rewrite our towers $K_{n}$ and $L_{n}$ to include $\eta_{i}^{*}$ (which now satisfies property (b) above) and $\varphi\left(\eta_{i}^{*}\right)$.

3. Main lemma. In this section we will prove the following result, which will be the main tool in proving the theorems discussed in the introduction. 
LEMMA 1. If the quadruple $\left(K_{0}, L_{0}, \varphi_{0}, \mathcal{E}\right)$ satisfies an elementary relation defined by $\left(K_{n}, L_{n}, \varphi\right)$ then there is an element $R, R \neq 0$ or 1 , in the valuation ring of $\varphi$ such that $\varphi$ is defined on $R$ with respect to some algebraic extension $E$ of $K_{0}$ and either $R$ is an elementary integral with respect to $E$, in which case $\varphi(R)=0$ or $R$ is an exponential of an elementary integral with respect to $E$, in which case $\varphi(R)=1$.

The proof relies on the following two propositions.

Proposition 3. Assume that $\left(K_{n}, L_{n}, \varphi\right)$ defines an elementary relation for $\left(K_{0}, L_{0}, \varphi_{0}, \mathcal{E}\right)$. Let $\eta_{j}$ be the last element among the $\left\{\eta_{i}\right\}$ such that $\eta_{j}$ is transcendental over $K_{j-1}=K_{0}\left(\eta_{1}, \ldots, \eta_{j-1}\right)$. If there is no $H$ in $K_{n}$, algebraic over $K_{j-1}$, such that $H \neq 0$ and $\varphi(H)=0$, then $\varphi\left(\eta_{j}\right)$ is algebraic over $L_{j-1}=L_{0}\left(\varphi\left(\eta_{1}\right), \ldots, \varphi\left(\eta_{j-1}\right)\right)$. Conversely, if for some $\eta_{k}$, transcendental over $K_{k-1}$, we have $\varphi\left(\eta_{k}\right)$ algebraic over $L_{k-1}$, then there is an $R, R \neq 0$, in $K_{k}$ such that $\varphi(R)=0$.

Proof. First, assume there is no $H$ in $K_{n}$ algebraic over $K_{j-1}$ such that $\varphi(H)=0$ and $H \neq 0$. Let $R, R \neq 0$, be an element of $K_{n}$ such that $\varphi(R)=$ 0 . Since $R$ is algebraic over $K_{0}\left(\eta_{1}, \ldots, \eta_{j}\right)=K_{j}$, let $P(X)=a_{n} X^{n}+\cdots+$ $a_{0}$ be a polynomial of minimal degree over $K_{j}$ such that $P(R)=0$. We can assume that the $a_{i}$ 's are in $\theta$, the valuation ring of $\varphi$. Since $P(R)=0$ and $\varphi(R)=0$, we have $\varphi\left(a_{0}\right)=0$. Since the degree of $P$ is minimal, $a_{0} \neq 0$, so we can use $a_{0}$ for our $R$. So now assume $R$ is in $K_{j}$. Write $R=R_{1} / R_{2}$ with $R_{1}, R_{2}$ in $K_{j-1}\left[\eta_{j}\right]$. Our initial assumption on $K_{j-1}$ implies $K_{j-1} \subset \mathcal{O}$ and so $R_{1}$ and $R_{2}$ are in $\theta$. Therefore, $\varphi\left(R_{1}\right)=0$ and $R_{1} \neq 0$. Let $R_{1}=b_{m} \eta_{j}^{m}+\cdots+b_{0}$ with each $b_{i}$ in $K_{j-1}$. Since some $b_{i}$ is nonzero, we have $\varphi\left(b_{i}\right) \neq 0$ for some $i$. Let $P_{0}(X)=\varphi\left(b_{m}\right) X^{m}+\cdots+\varphi\left(b_{0}\right)$. As we have just seen, this polynomial is not identically zero. Since $\varphi\left(R_{1}\right)=0$, we have $\varphi\left(R_{1}\right)=P_{0}\left(\varphi\left(\eta_{j}\right)\right)=0$ so $\varphi\left(\eta_{j}\right)$ is algebraic over $L_{j-1}$.

Conversely, assume that for some $k, \eta_{k}$ is not algebraic over $K_{k-1}$ while $\varphi\left(\eta_{k}\right)$ is algebraic over $L_{k-1}$. Let $S_{0}(X)$ be a nonzero polynomial in $L_{k-1}[X]$ such that $S_{0}\left(\varphi\left(\eta_{j}\right)\right)=0$. Let $S(X)$ be a polynomial in $K_{k-1}[X]$ whose coefficients are mapped to the coefficients of $S_{0} . S\left(\eta_{k}\right) \neq 0$ since $\eta_{k}$ is transcendental over $K_{k-1}$ while $\varphi\left(S\left(\eta_{k}\right)\right)=0 . R=S\left(\eta_{k}\right)$ gives our desired $R$.

Proposition 4. Let $k$ be $a$ differential field and $K a$ differential extension field with the same constants as $k$, which is furthermore algebraic over $k(t)$ for some $t$ in $K$. Suppose that $c_{1}, \ldots, c_{n}$ are constants which are linearly independent over $\mathbf{Q}$ and $u_{1}, \ldots, u_{n}, v \in K\left(u_{1} \neq 0\right.$ for all $\left.i\right)$. Suppose further that for each derivation $D$ of $K$ we have $\Sigma c_{i} D u_{i} / u_{i}+D v$ is in $k$. If for each derivation $D$ of $K$, we have $D t \in k$, then $u_{1}, \ldots, u_{n}$ are in $\bar{k}$ (the algebraic closure of $k$ in $K)$ and $v=c t+d$, where $c$ is a constant and $d$ is in $\bar{k}$. If for each $D$, 
$D t / t \in k$, then we have $v \in \bar{k}$ and there are $\nu_{0}, \nu_{1}, \ldots, \nu_{n}$ in $\mathbf{Z}, \nu_{0} \neq 0$ s.t. $u_{i}^{\nu_{0}} / t^{\nu_{i}} \in \bar{k}$ for $1 \leqslant i \leqslant n$.

This proposition is proven in [8].

Proof of LemMa 1. The idea of the proof is very simple. Proposition 3 tells us that $\left(K_{n}, L_{n}, \varphi\right)$ defines an elementary relation if and only if there is an $\eta_{i}$ transcendental over $K_{i-1}$ such that $\varphi\left(\eta_{i}\right)$ is algebraic over $L_{i-1}$. Proposition 4 tells us "how" it is algebraic over $L_{i-1}$ and allows us to construct an $R$ of the desired form. The proof proceeds by an induction on the transcendence degree of $K_{n}$ over $K_{0}$.

If there exists an $R$ in $K_{n}$ such that $R$ is algebraic over $K_{0}, R \neq 0$, $\varphi(R)=0$, then $R$ satisfies the conclusion of the Lemma since it is an elementary integral with respect to $K_{0}(R)$. In particular, if $K_{n}$ is algebraic with respect to $K_{0}$, such an $R$ exists.

Let the transcendence degree of $K_{n}$ over $K_{0}$ be 1 . By the remark of the preceding paragraph, we need only consider the case that there exists no $R$ in $K_{n}$ with $R$ algebraic over $K_{0}, R \neq 0, \varphi(R)=0$. Therefore, by Proposition 3, there is some $\eta_{i}$, transcendental over $K_{0}$, such that $\eta_{i}$ is an elementary integral or exponential of an elementary integral with respect to some algebraic extension $E$ of $K_{0}$ and $\varphi\left(\eta_{i}\right)$ is algebraic over $L_{0}$. If $\eta_{i}$ is an elementary integral with respect to $E$, then Property 2 of elementary relations tells us that $\varphi\left(\eta_{i}\right)$ is an elementary integral with respect to $\varphi(\theta \cap E)$. Since $\varphi\left(\eta_{i}\right)$ is algebraic over $L_{0}$, and so over $\varphi(\theta \cap E)$, the Kolchin-Ostrowski Theorem (with $F=\varphi(\theta \cap E)$ ) tells us that $\varphi\left(\eta_{i}\right)$ actually is in $\varphi(\theta \cap E)$. Pick $A$ in $E$ such that $\varphi(A)=\varphi\left(\eta_{i}\right)$ and let $R=\eta_{i}-A . R \neq 0$ or 1 , for otherwise $\eta_{i}$ would be algebraic over $K_{0} . R$ is an elementary integral with respect to $E$ and $\varphi(R)=0$ so $R$ satisfies the conclusion of the Lemma. If $\eta_{i}$ is an exponential of an elementary integral with respect to $E$, then $\varphi\left(\eta_{i}\right)$ is an exponential of an elementary integral with respect to $\varphi(\theta \cap E)$ and furthermore $\varphi\left(\eta_{i}\right) \neq 0$. Using the Kolchin-Ostrowski Theorem again, we can find an $m \neq 0$ in $\mathbf{Z}$ such that $\varphi\left(\eta_{i}\right)^{m}$ is in $\varphi(\theta \cap E)$. Let $A$ be an element of $E$ such that $\varphi(A)=\varphi\left(\eta_{i}\right)$ and let $R=\eta_{i}^{m} / A . R \neq 0$ or 1 for otherwise $\eta_{i}$ would be algebraic over $K_{0} . R$ is an exponential of an elementary integral with respect to $E$ and $\varphi(R)=1$. to $E$ and $\varphi(R)=1$.

If the transcendence degree of $K_{n}$ over $K_{0}$ is $>2$, we are going to show how to find subtowers $\tilde{K}_{n}$ of $K_{n}$ and $\tilde{L}_{n}$ of $L_{n}$ such that when we restrict $\varphi$ to $\tilde{K}_{n}$ we are still defining an elementary relation and such that the transcendence degree of $\tilde{K}_{n}$ over $K_{0}$ is less than that of $K_{n}$ over $K_{0}$. Our induction hypothesis will then give us our result. To do this it is sufficient to show the following: Assume we are given: 
(1) $K$ and $L$ differential fields with sets of derivations $\mathscr{D}$ and $\Delta$, and a subset $\mathcal{E}$ of $\mathscr{D}$;

(2) generalized elementary extensions $K\left(\zeta_{1}, \alpha_{1}, \zeta_{2}, \alpha_{2}\right), L\left(\xi_{1}, \beta_{1}, \xi_{2}, \beta_{2}\right)$ of $K$ and $L$ such that:

(a) $\zeta_{1}, \zeta_{2}$ are algebraically independent over $K$,

(b) $\zeta_{1}$ is an elementary integral or an exponential of an elementary integral over $K, \alpha_{1}$ is algebraic over $K\left(\zeta_{1}\right), \zeta_{2}$ is an elementary integral or an exponential of an elementary integral over $K\left(\zeta_{1}, \alpha_{1}\right)$ and $\alpha_{2}$ is algebraic over $K\left(\zeta_{1}, \alpha_{1}, \zeta_{2}\right) . \xi_{1}, \beta_{1}, \xi_{2}, \beta_{2}$ play the same role with respect to $L$;

(3) a place $\varphi$, with valuation ring $\theta$ such that $\varphi\left(\zeta_{i}\right)=\xi_{i}$ and $\varphi\left(\alpha_{i}\right)=\beta_{i}$ such that $\left(K\left(\zeta_{1}, \alpha_{1}, \zeta_{2}, \alpha_{2}\right), L\left(\xi_{1}, \beta_{1}, \xi_{2}, \beta_{2}\right), \varphi\right)$ defines an elementary relation for $\left(K, L,\left.\varphi\right|_{K}, \mathcal{E}\right)$.

We then must be able to find elements $\tilde{\alpha}_{1}, \tilde{\zeta}, \tilde{\alpha}_{2}$ in $K\left(\zeta_{1}, \alpha_{1}, \zeta_{2}, \alpha_{2}\right)$ and $\tilde{\beta}_{1}$, $\tilde{\xi}, \tilde{\beta}_{2}$ in $L\left(\xi_{1}, \beta_{1}, \xi_{2}, \beta_{2}\right)$ such that:

(1) $\tilde{\alpha}_{1}$ is algebraic over $K, \tilde{\xi}$ is an elementary integral or exponential of an elementary integral with respect to $K\left(\tilde{\alpha}_{1}\right), \tilde{\alpha}_{2}$ algebraic over $K\left(\tilde{\alpha}_{1}, \tilde{\zeta}\right)$ and $\tilde{\beta}_{1}$, $\tilde{\xi}, \tilde{\beta}_{2}$ play the same roles with respect to $L$, and

(2) $\left(K\left(\tilde{\alpha}_{1}, \tilde{\zeta}, \tilde{\alpha}_{2}\right), L\left(\tilde{\beta}_{1}, \tilde{\xi}, \tilde{\beta}_{2}\right), \tilde{\varphi}\right)$ defines an elementary relation for $(K, L$, $\varphi, \mathcal{E})$ where $\tilde{\varphi}$ is the restriction of $\varphi$ to $K\left(\tilde{\alpha}_{1}, \tilde{\zeta}, \tilde{\alpha}_{2}\right)$.

Assuming we have shown that such a construction exists, we can take a tower $K_{n}$ such that the transcendence degree of $K_{n}$ over $K_{0}$ is $>2$, let $\zeta_{1}=\eta_{k}$, $\zeta_{2}=\eta_{j}$ (where $\eta_{k}$ and $\eta_{j}$ are the last two $\eta_{i}$ 's to be algebraically independent over $\left.K_{0}\left(\eta_{1}, \ldots, \eta_{k-1}\right)\right), K=K_{0}\left(\eta_{1}, \ldots, \eta_{k-1}\right)$. Pick $\alpha_{1}$ such that $K_{0}\left(\eta_{1}, \ldots, \eta_{k}, \alpha_{1}\right)=K_{0}\left(\eta_{1}, \ldots, \eta_{j-1}\right)$ and $\alpha_{2}$ such that $K_{0}\left(\eta_{1}, \ldots, \eta_{j}, \alpha_{2}\right)=$ $K_{n}$. Let $\xi_{1}=\varphi\left(\eta_{k}\right), \quad \beta_{1}=\varphi\left(\alpha_{1}\right), \quad \xi_{2}=\varphi\left(\eta_{j}\right), \quad \beta_{2}=\varphi\left(\alpha_{2}\right)$ and $L=$ $L_{0}\left(\varphi\left(\eta_{1}\right), \ldots, \varphi\left(\eta_{k-1}\right)\right)$. Now apply the construction to find a tower of smaller transcendence degree.

To proceed with the construction: Note that we can assume that if $u \neq 0$ is algebraic over $K\left(\zeta_{1}\right)$, then $u \in \mathcal{O}$ and $\varphi(u) \neq 0$. If $u \notin \theta$ then $u^{-1}$ would be in $\theta$ and $\varphi\left(u^{-1}\right)=0$. We could then take $\tilde{\alpha}_{1}=0, \tilde{\zeta}=\zeta_{1}, \alpha_{2}=u, \tilde{\beta}_{1}=0$, $\tilde{\xi}=\xi$, and $\tilde{\beta}_{2}=0$, and we would be done. If $u \in \theta$ and $\varphi(u)=0$, we could make the same choices and be done.

We now have four cases to consider, corresponding to $\zeta_{1}$ and $\zeta_{2}$ being elementary integrals or exponentials of elementary integrals.

Case 1. Both $\zeta_{1}$ and $\zeta_{2}$ are exponentials of elementary integrals. Say

$$
\frac{D \zeta_{1}}{\zeta_{1}}=\sum c_{i} \frac{D U_{i}}{U_{i}}+D V, \quad \frac{D \zeta_{2}}{\zeta_{2}}=\sum d_{i} \frac{D S_{i}}{S_{i}}+D T
$$

for some choice of $U_{1}, \ldots, U_{n}, V \in K$ and $S_{1}, \ldots, S_{m}, T$ in $K\left(\zeta_{1}, \alpha_{1}\right)$ and where $\left\{1, c_{1}, \ldots, c_{n}\right\},\left\{1, d_{1}, \ldots, d_{m}\right\}$ are Q-linearly independent sets of constants. Let $u_{i}=\varphi\left(U_{i}\right), v=\varphi(V), s_{i}=\varphi\left(S_{i}\right), t=\varphi(T)$. By our assumptions about $\varphi$, we have 


$$
\frac{\delta \xi_{1}}{\xi_{1}}=\sum c_{i} \frac{\delta u_{i}}{u_{i}}+\delta v \text { and } \frac{\delta \xi_{2}}{\xi_{2}}=\sum d_{i} \frac{\delta s_{i}}{s_{i}}+\delta t
$$

for all $\delta \in \Delta$. Since $\xi_{2}$ is algebraic over $L\left(\xi_{1}\right), \delta \xi_{1} / \xi_{1} \in L$ and $\delta \xi_{2} / \xi_{2}-$ $\left(\sum d_{i} \delta s_{i} / s_{i}+\delta t\right)=0 \in L$, we can let the $k$ of Proposition 4 be $L$ and the $K$ of Proposition 4 be $L\left(\xi_{1}, \beta_{1}, \xi_{2}, \beta_{2}\right)$ and get that there exist $\nu, \nu_{0}, \nu_{1}, \ldots, \nu_{m} \in \mathbf{Z}$, $\nu \neq 0$, s.t. $\xi_{2}^{\nu} / \xi_{1}^{\nu_{0}}, s_{1}^{\nu} / \xi_{1}^{\nu_{1}}, \ldots, s_{m}^{\nu} / \xi_{1}^{\nu_{m}}$ are all algebraic over $L$ and $t$ is algebraic over $L$.

I claim that $S_{1}^{p} / \zeta_{1}^{\nu_{1}}, \ldots, S_{m}^{p} / \zeta_{1}^{p_{m}}$, are all algebraic over $K$. Let $H_{i}(X)$ be the minimum polynomial of $s_{i}^{p} / \xi_{1}^{p_{1}}$ in $L[X]$. Pull each $H_{i}$ back via $\varphi$ to a polynomial $H_{i}^{*}(X)$ in $K[X]$. Then each element $H_{i}^{*}\left(S_{i}^{\nu} / \zeta_{i}^{*}\right)$ is algebraic over $K\left(\zeta_{1}\right)$ and $\varphi\left(H_{i}^{*}\left(S_{i}^{\nu} / \zeta_{1}^{\nu}\right)\right)=0$, so by the above remark $H_{i}^{*}\left(S_{i}^{\nu} / \zeta_{1}^{p_{1}}\right)=0$ for each $i$. Therefore, each $S_{i}^{\nu} / \zeta_{1}^{\nu_{1}}$ is algebraic over $K$. Similarly one can show $T$ is algebraic over $K$.

Since our fields are all of characteristic 0 , we can find $\tilde{\alpha}_{1}$ (by the primitive element theorem) s.t. $\tilde{\alpha}_{1} \in \mathcal{O}$ and $K\left(T, S_{1}^{\nu} / \zeta_{1}^{\nu_{1}}, \ldots, S_{n}^{\nu} / \zeta_{1}^{\nu_{n}}\right)=K\left(\tilde{\alpha}_{1}\right)$. Let $\tilde{\zeta}=\zeta_{2}^{\nu} / \zeta_{1}^{\nu_{0}}$; then $\tilde{\zeta}$ is an exponential of an elementary integral since

$$
\begin{aligned}
D \tilde{\zeta} / \tilde{\zeta}= & D\left(\zeta_{2}^{\nu} / \zeta_{1}^{\nu_{0}}\right) /\left(\zeta_{2}^{\nu} / \zeta_{1}^{\nu_{0}}\right)=\sum d_{i} \frac{D\left(S_{i}^{\nu} / \zeta_{1}^{\nu_{i}}\right)}{S_{i}^{\nu} / \zeta_{1}^{\nu_{i}}} \\
& +\nu D T-\left(\nu_{0}+\sum d_{i} \nu_{i}\right)\left(\sum c_{i} \frac{D U_{i}}{U_{i}}+D V\right) .
\end{aligned}
$$

Let $\tilde{\alpha}_{2}=0$ and $\tilde{\beta}_{1}=\varphi\left(\tilde{\alpha}_{1}\right)$ (note that this implies that $s_{i}^{p} / \xi_{1}^{p_{i}} \in L\left(\tilde{\beta}_{1}\right)$ since everything algebraic over $K\left(\zeta_{1}\right)$ is in $\mathcal{O}$ so if $S_{i}^{p} / \zeta_{1}^{\nu_{l}}=\Sigma_{j=0}^{l} a_{j} \tilde{\alpha}_{1}^{j}$ with each $a_{j} \in K$, then $\left.s_{i}^{\nu} / \xi_{1}^{\nu_{i}}=\varphi\left(S_{i} / \zeta_{1}^{\nu^{\prime}}\right)=\Sigma \varphi\left(a_{i}\right) \tilde{\beta}_{1}^{j}\right)$. Let $\tilde{\xi}=\xi_{2}^{\nu} / \xi_{1}^{\nu_{0}}$ and $\tilde{\beta}_{2}=0$. Let $\tilde{\varphi}$ be the restriction of $\varphi$ to $K\left(\tilde{\alpha}_{1}, \tilde{\zeta}\right)$ and $\tilde{\mathcal{\theta}}=\hat{\theta} \cap K\left(\tilde{\alpha}_{1}, \tilde{\zeta}\right)$. $\tilde{\varphi}$ satisfies conditions (1) through (3) of $\S 2$, as can be easily checked. Finally, $\tilde{\zeta}$ is transcendental over $K\left(\tilde{\alpha}_{1}\right)$ (otherwise $\zeta_{1}$ and $\zeta_{2}$ would be algebraically dependent), while $\varphi(\tilde{\zeta})=\tilde{\xi}=\xi_{2}^{p} / \xi_{1}^{\nu_{0}}$ is algebraic over $L\left(\tilde{\beta}_{1}\right)$. Therefore, by the second part of Proposition 3, there exists a $\tilde{R}$ in $K\left(\tilde{\alpha}_{1}, \tilde{\zeta}\right)$ s.t. $\varphi(\tilde{R})=0$ and $D \tilde{R} \neq 0$.

The methods used in the next three cases are the same as those used in Case 1 . I will therefore only outline the steps.

Case 2. Both $\zeta_{1}$ and $\zeta_{2}$ are elementary integrals. Let

$$
D \zeta_{1}=\sum c_{i} \frac{D U_{i}}{U_{i}}+D V, \quad D \zeta_{2}=\sum d_{i} \frac{D S_{i}}{S_{i}}+D T,
$$

for all $D$ in $\Phi$. Let

$$
\delta \xi_{1}=\sum c_{i} \frac{\delta u_{i}}{u_{i}}+\delta v, \quad \delta \xi_{2}=\sum d_{i} \frac{\delta s_{i}}{s_{i}}+\delta t
$$


for all $\delta$ in $\Delta$. We can assume again that $\xi_{2}$ is algebraic over $L\left(\xi_{1}\right)$ and so by Proposition 4, the $s_{i}$ 's are algebraic over $L$ and $\xi_{2}-t=c \xi_{1}+d$ for some $c$ (a constant) and $d$ algebraic over $L$. In the same manner as Case 1 , we can show that all the $S_{i}$ 's are algebraic over $K$. Let $\tilde{\alpha}_{1}$ be an element s.t. $K\left(\tilde{\alpha}_{1}\right)=$ $K\left(S_{1}, \ldots, S_{n}\right)$ and let $\tilde{\zeta}=\zeta_{2}-c \zeta_{1}-T$.

$$
D \tilde{\zeta}=\sum d_{i} \frac{D S_{i}}{S_{i}}-c \sum c_{i} \frac{D U_{i}}{U_{i}}-c D V
$$

so $\tilde{\zeta}$ is an elementary integral over $K\left(\tilde{\alpha}_{1}\right)$. Let $\beta_{1}=\varphi\left(\tilde{\alpha}_{1}\right), \tilde{\xi}=\xi_{2}-c \xi_{1}-t=$ $d$ which is algebraic over $L$, so again we can get a $\tilde{R}$ in $K\left(\tilde{\alpha}_{1}, \zeta\right)$ s.t. $\varphi(\tilde{R})=0$ while $\tilde{R} \neq 0$.

Case 3. $\zeta_{1}$ is an exponential of an elementary integral and $\zeta_{2}$ is an elementary integral. Let

$$
\begin{array}{ll}
\frac{D \zeta_{1}}{\zeta_{1}}=\sum c_{i} \frac{D U_{i}}{U_{i}}+D V, & D \zeta_{2}=\sum d_{i} \frac{D S_{i}}{S_{i}}+D T, \\
\frac{\delta \xi_{1}}{\xi_{1}}=\sum c_{i} \frac{\delta u_{i}}{u_{i}}+\delta v, & \delta \zeta_{2}=\sum d_{i} \frac{\delta s_{i}}{s_{i}}+\delta t .
\end{array}
$$

As before, we can assume $\xi_{2}$ is algebraic over $L\left(\xi_{1}\right)$ and get, using Proposition 4 , that $s_{i}{ }^{\prime} / \xi_{1}^{p_{1}}$ and $\xi_{2}-t$ are algebraic over $L$. This allows us to assume that $\left\{S_{i}^{p} / \zeta^{\nu_{1}}\right\}$ are algebraic over $K$. Let $\tilde{\alpha}_{1}$ be an element

$$
\begin{aligned}
& \text { s.t. } K\left(S_{1}^{\nu} / \zeta_{1}^{\nu_{1}}, \ldots, S_{n}^{\nu} / \zeta_{1}^{\nu_{n}}\right)=K\left(\tilde{\alpha}_{1}\right), \quad \tilde{\zeta}=\nu\left(\zeta_{2}-T\right) . \\
& D(\tilde{\zeta})=\sum d_{i} \frac{D\left(S_{i}^{\nu} / \zeta_{1}^{\nu_{i}}\right)}{S_{i}^{\nu} / \zeta_{1}^{\nu_{i}}}-\left(\sum \nu_{i} d_{i}\right)\left(\sum c_{i} \frac{D U_{i}}{U_{i}}+D V\right)
\end{aligned}
$$

so $\tilde{\zeta}$ is an elementary integral over $K$. Now proceed as above to get $\tilde{R}, \tilde{\beta}_{1}, \tilde{\beta}_{2}$, $\tilde{\xi}, \tilde{\varphi}$.

Case 4. $\zeta_{1}$ is an elementary integral and $\zeta_{2}$ is an exponential of an elementary integral. Let $D \zeta_{1}=\Sigma c_{i} D U_{i} / U_{i}+D V, D \zeta_{2} / \zeta_{2}=\Sigma d_{i} D S_{i} / S_{i}+$ $D T . \delta \xi_{1}, \delta \xi_{2}$ similarly. Proceeding as before, we get $\xi_{2},\left\{s_{i}\right\}$ algebraic over $L$ and $t=c \xi_{1}+b$, for some $c$, a constant and $b$ algebraic over $L$. One can then show that the $S_{i}$ and $T-c \zeta_{1}$ are algebraic over $L$. Let $\tilde{\alpha}_{1}$ be s.t. $K\left(\tilde{\alpha}_{1}\right)=$ $K\left(S_{1}, \ldots, S_{l}, T-c \zeta\right)$ and $\tilde{\zeta}=\zeta_{2}$.

$$
\begin{aligned}
\frac{D \zeta_{2}}{\zeta_{2}} & =\sum d_{i} \frac{D S_{i}}{S_{i}}+D T \\
& =\sum d \frac{D S_{i}}{S_{i}}+c\left(\sum c_{i} \frac{D U_{i}}{U_{i}}+D V\right)+D\left(T-c \zeta_{1}\right) .
\end{aligned}
$$

So $\zeta_{2}$ is an exponential of an elementary integral over $K\left(\tilde{\alpha}_{1}\right)$. Now proceed as before to get $\tilde{R}, \tilde{\beta}_{1}, \tilde{\beta}_{2}, \tilde{\xi}$. 
4. Ritt's theorem: integrals satisfying elementary relations. In this section we will prove

THEOREM 1. Let $E$ be an ordinary differential field, with derivation ', and $E\left(w_{1}, \ldots, w_{n}\right)$ a differential extension with the same field of constants, such that $w_{i}^{\prime} \in E$. If the integrals $w_{1}, \ldots, w_{n}$ satisfy an elementary relation, then some nontrivial linear combination with constant coefficients lies in an elementary extension of $E$.

Proof. Let $K_{0}, L_{0}, \varphi_{0}, \mathcal{Q}$ and $\mathcal{E}$ be as in example (a) of $\S 2$ and let $\left(K_{m}, L_{m}\right.$, $\varphi)$ define an elementary relation for $\left(K_{0}, L_{0}, \varphi_{0}, \mathcal{E}\right)$. Let $R$ be the element of $K_{m}$ whose existence is guaranteed by Lemma 1 . If $R$ is algebraic over $K_{0}$, taking $G$ to be $R$ or $R-1$, we can assume $\varphi(G)=0$ and $D G \neq 0$ for some $D$ in $\delta$. Let $H(X)=a_{m} X^{m}+\cdots+a_{0}$ be a minimal polynomial of $G$ in $E\left[W_{1}, \ldots, W_{n}\right][X]$; then $\varphi(H(G))=\varphi\left(a_{0}\right)=0$. By the minimality of $H$, we know that $a_{0} \neq 0$. Let $a_{0}=F_{n}\left(W_{1}, \ldots, W_{n}\right)+\cdots+F_{0}$ where the $F_{i}$ 's are forms of degree $i$ over $E, n \geqslant 1$ and $F_{n}\left(W_{1}, \ldots, W_{n}\right) \neq 0$. Since $\varphi\left(a_{0}\right)=$ $F_{n}\left(w_{1}, \ldots, w_{n}\right)+\cdots+F_{0}$, the $w_{i}$ are algebraically dependent over $E$. Therefore, by the Kolchin-Ostrowski Theorem, we know some nontrivial linear combination with constant coefficients is in $E$, and so satisfies the conclusion of the theorem.

Now assume $R$ is not algebraic over $K_{0}$ and that $D R / R=\sum_{i=1}^{l} c_{i} D U_{i} / U_{i}$ $+D V$ for all $D$ in $D$, where the $U_{i}$ and $V$ are algebraic over $E\left(W_{1}, \ldots, W_{n}\right)$ and $\left\{c_{1}, \ldots, c_{n}\right\}$ are linearly independent over $\mathbf{Q}$. Let $\varphi\left(U_{i}\right)=u_{i}$ and $\varphi(V)$ $=v$, then $0=\sum_{i=1}^{l} c_{i} u_{i} / u_{i}+v^{\prime}$. If we let $k=E\left(w_{1}, \ldots, w_{n-1}\right)$ and $t=w_{n}$ and apply Proposition 4 to $\sum_{i=1}^{l} c_{i} u_{i} / u_{i}+v^{\prime}$ we get that $u_{1}, \ldots, u_{n}$ are algebraic over $E\left(w_{1}, \ldots, w_{n-1}\right)$ and $v=a_{n} w_{n}+b_{n}$ where $a_{n}$ is a constant and $b_{n}$ is algebraic over $E\left(w_{1}, \ldots, w_{n-1}\right)$. Applying this proposition to $\sum_{i=1}^{l} c_{i} u_{i}^{\prime} / u_{i}+b_{n}^{\prime}=-a_{n} w_{n}^{\prime} \in E$, where $k$ is now $E\left(w_{1}, \ldots, w_{n-2}\right), t=$ $w_{n-1}$, we get the $u_{i}$ algebraic over $E\left(w_{1}, \ldots, w_{n-2}\right)$ and $b_{n}=a_{n-1} w_{n-1}+$ $b_{n-1}$ with $a_{n-1}$ a constant and $b_{n-1}$ algebraic over $E\left(w_{1}, \ldots, w_{n-2}\right)$. Continuing in this way, we get that there exist constants $a_{1}, \ldots, a_{n}$ such that $a_{1} w_{1}^{\prime}+\cdots+a_{n} w_{n}^{\prime}=\sum c_{i} u_{i}^{\prime} / u_{i}+b^{\prime}$ where the $u_{i}$ and $b$ are algebraic over $E$. If some $a_{i}$ is nonzero then we have a nontrivial linear combination of the $w_{i}$, with constant coefficients, which is an elementary integral and therefore lies in an elementary extension of $E$. If all the $a_{i}$ 's are 0 , we will get a contradiction. All the $a_{i}$ 's being zero implies $v$ is algebraic over $E$. For each $i$ let $H_{i}(X)$ be a polynomial in $E[X]$ such that $H_{i}\left(u_{i}\right)=0$ and $H(X)$ a polynomial in $E[X]$ such that $H(v)=0 . \varphi$ is an isomorphism on $E$, so $\varphi\left(H_{i}\left(U_{i}\right)\right)=$ $\varphi(H(V))=0$ for each $i$. If $H_{i}\left(U_{i}\right) \neq 0$ for some $i$ or $H(V) \neq 0$, then we would be reduced to the case where we have a $G \neq 0$ algebraic over $K_{0}$ such that $\varphi(G)=0$, and this was treated above. Therefore $H_{i}\left(U_{i}\right)=0$ for all $i$ and 
$H(V)=0$, and so the $U_{i}$ and $V$ are algebraic over $E$. Since $D(E)=0$ for all $D$ in $\mathcal{E}$, we get, for all $D$ in $\mathcal{E}, D\left(U_{i}\right)=0$ for all $i$ and $D(V)=0$. Therefore $D(R)=0$ for all $D$ in $\mathcal{E}$. Since $\varphi(R)=1$, Property 3 of elementary relations, applied to $R-1$ tells us that $R=1$, a contradiction. If $R$ is an elementary integral with respect to $E$, the proof is the same.

We have actually shown more than the theorem stated above. We have shown that if $w_{1}, \ldots, w_{n}$ satisfy an elementary relation, then there exist constants $c_{1}, \ldots, c_{n}$, not all zero, constants $d_{1}, \ldots, d_{l}$ and elements $u_{1}, \ldots, u_{n}, v$, algebraic over $E$ such that

$$
c_{1} w_{1}^{\prime}+\cdots+c_{n} w_{n}^{\prime}=\sum_{i=1}^{l} d_{i} \frac{u_{i}^{\prime}}{u_{i}}+v^{\prime} .
$$

Taking the trace of both sides of the equation, with respect to $E$, we get, for some integer $n$,

$$
\begin{aligned}
n\left(c_{1} w_{1}^{\prime}+\cdots+c_{n} w_{n}^{\prime}\right) & =\sum_{i=1}^{l} d_{i} \operatorname{Trace}\left(\frac{u_{i}^{\prime}}{u_{i}}\right)+\text { Trace } v^{\prime} \\
& =\sum_{i=1}^{l} d_{i} \frac{\left(\operatorname{Norm} u_{i}\right)^{\prime}}{\operatorname{Norm} u_{i}}+(\text { Trace } v)^{\prime}
\end{aligned}
$$

since any algebraic automorphism is automatically a differential automorphism. Therefore, we can conclude that if $w_{1}, \ldots, w_{n}$ satisfy an elementary relation, there are constants $c_{1}, \ldots, c_{n}$, not all zero, constants $d_{1}, \ldots, d_{l}$, and elements $u_{1}, \ldots, u_{n}, v$ in $E$ such that

$$
c_{1} w_{1}^{\prime}+\cdots+c_{n} w_{n}^{\prime}=\sum_{i=1}^{l} d_{i} \frac{u_{i}^{\prime}}{u_{i}}+v^{\prime}
$$

This fact also follows from Liouville's Theorem and will be useful in computing examples.

I now want to make the connection between the above theorem and the theorem that Ritt actually proved. Ritt restricted himself to considering integrals of elementary functions. Let $f(z)$ be such a function and assume that we have restricted its domain $\& \subset \subset \mathbf{C}$ s.t. $f$ and the ancillary functions used to define the elementary tower to which $f$ belongs, are analytic in $\mathbb{B S}$. In this case $\int f(t) d t$ is an analytic function. Let its graph in $\& \times \mathbf{C}$ be denoted by $\pi$. Ritt's theorem says: If, at each point of an uncountable subset of $\mathfrak{R}$, an elementary function of two variables $F(w, z)$ not identically 0 on $\&$ \& $\times \mathbf{C}$ either assumes the value zero or has a singularity, then $\int f d t$ is an elementary function of $z$. Assuming one has such a function, Ritt shows [4, pp. 87-92] that we can assume we have the following situation: There is a region $\mho^{\prime} \subset \mathbf{C}^{2}$ and an elementary tower $K_{n} \supset \cdots \supset K_{0}=\mathbf{C}\left(z\right.$, w) s.t. (1) $K_{i}=$ $K_{i-1}\left(\eta_{i}\right)$ where $\eta_{i}$ is analytic in (S' and is either a logarithm, exponential or 
algebraic over $K_{i-1}$, (2) there is an $F$ in $K_{n}$ s.t. $\partial F / \partial w \neq 0$ and $F$ is zero on IL $\cap \mathbb{S}^{\prime}$ (which is still uncountable). We can translate this into our algebraic language in the following way. Let us first assume that $\left\{K_{n}\right\}$ is a tower minimal with respect to having the properties stated above. Let $k=$ $\mathbf{C}(z, w)\langle f\rangle$-the smallest differential ' field containing! $\mathbf{C}(z, w)$ and $f$. Let $K_{0}^{*}=k$ and $K_{i}^{*}=K_{i-1}^{*}\left(\eta_{i}\right) . K_{n}^{*}$ is a field of functions of two variables meromorphic in (S'. If $G \in K_{n}^{*}$, the set of points, $P(G)$, where $G$ is infinite or undefined is a subvariety of complex dimension $1[2 \mathrm{a}, \mathrm{p}$. 247]. Since $\mathfrak{N}$ is the graph of an analytic function, it is an irreducible variety, also of dimension 1 , and so $P(G) \cap\left(\Re \cap\left(S^{\prime}\right)\right.$ is either a finite set of points or all of $\Re \cap \mathbb{S}^{\prime}$ [2a]. Let $\theta=\left\{G \mid G\right.$ is defined and finite at some point of $\left.\mathscr{N} \cap \mathbb{F S}^{\prime}\right\}$ and let $I=\left\{G \in O \mid G(x)=0\right.$ for all $x \in \mathfrak{N} \cap \mathbb{S}^{\prime}$ for which $G$ is defined $\}$. Using the above, we see that $\theta$ is a valuation ring with maximal ideal equal to $i$. Define $\varphi$ on $\theta$ as $\varphi(G(z, w))=G(z, w(z))$ where $w(z)=\int f(z) d z$. This maps $\theta$ onto a field of meromorphic functions on $\& \subset \mathbf{C}$. Consider the tower of fields

$$
\begin{aligned}
L_{n} & =L_{n-1}\left(\varphi\left(\eta_{n}\right)\right) \supset \cdots \supset L_{1}=L_{0}\left(\varphi\left(\eta_{1}\right)\right) \supset L_{0} \\
& =\mathbf{C}(z)\langle f(z), w(z)\rangle .
\end{aligned}
$$

$L_{n}$ is a differential field. Each $\eta_{i}$ is an exponential or logarithm or algebraic with respect to $K_{i-1}^{*}$. If $\eta_{i}=e^{f_{i}(z, w)}$ then $f_{i}(z, w) \in \mathcal{O}$, for otherwise $f_{i}^{-1}$ would be in the maximal ideal of $\theta$ and we would have an elementary relation in a smaller tower. Similarly, we can check out the other properties $\varphi$ must have. Since there is an $F$ in $K_{n}^{*}$ s.t. $F$ is zero on ' $\mathscr{S}^{\prime} \cap \mathscr{N}$, we have $\varphi(F)=0$, so $\left(K_{n}^{*}\right.$, $\left.L_{n}, \varphi\right)$ is an elementary relation for $(\mathbf{C}(z, w)\langle f\rangle, \mathbf{C}(z)\langle f, w(z)\rangle, \varphi,\{\partial / \partial w\})$. We therefore see that Ritt's theorem is a special case of Theorem 1 .

Finally, I want to consider some applications of Theorem 1.

(a) Elliptic integrals. Let $\mathbf{C}\left(z, y_{1}, \ldots, y_{n}\right)$ be an algebraic extension of $\mathbf{C}(z)$, where for each $i, y_{i}^{2}=f_{i}(z)$, a cubic polynomial with no repeated roots. Consider this field as a differential field with derivation 's.t. $z^{\prime}=1$. Assume further that

$$
\left[\mathbf{C}\left(z, y_{1}, \ldots, y_{n}\right): \mathbf{C}\left(z, y_{1}, \ldots, \hat{y}_{i}, \ldots, y_{n}\right)\right]=2 \text { for all } i .
$$

I will show that in this case, the elliptic integrals $\int 1 / y_{1} d z, \ldots, \int 1 / y_{n} d z$ satisfy no elementary relation.

In particular, if the $f_{i}$ 's have no common roots, the corresponding integrals satisfy no elementary relation. We will again use some facts about function fields of one variable, for which we refer to [2]. First, let $C \subset F \subset E$, where $E, F$ are function fields of one variable over their common field of constants $C$, and $[E: F]<\infty$. If $z$ and $y$ are elements of $F$ and $y d z$ is a differential of the first kind with respect to $F$, then $y d z$ is a differential of the first kind with 
respect to $E$ (one needs only to expand in local coordinates to check this out). In particular, $\left\{z / y_{i}\right\}$ are differentials of the first kind in $\mathbf{C}\left(z, y_{1}, \ldots, y_{n}\right)$. Now assume $\left\{\int d z / y_{i}\right\}$ satisfy an elementary relation. Then by Theorem 1 , we could find $u_{1}, \ldots, u_{n}, v \in \mathbf{C}\left(z, y_{1}, \ldots, y_{n}\right)$ and constants $\left\{c_{i}\right\},\left\{d_{i}\right\}$ s.t. $1 / y_{1}+\sum_{i=2}^{n} c_{i} / y_{i}=\sum d_{i} u_{i}^{\prime} / u_{i}+v^{\prime}$. Referring to the discussion at the end of $\S 2$, we can assume the $d_{i}$ 's are linearly independent over $\mathbf{Q}$. Let $P$ be a zero of some $u_{i}$. Taking residues at $P$, and noting that $\operatorname{Res}_{P}\left(d z / y_{1}+\Sigma c_{i} d z / y_{i}\right)=0$ $=\operatorname{Res}_{P}\left(v^{\prime} d z\right)$ and that $\operatorname{Res}_{P}\left(u_{i}^{\prime} / u_{i} d z\right) \in \mathbf{Z}$, we see that each $d_{i}=0$. Therefore,

$$
1 / y_{1}+\sum_{i=2}^{n} c_{i} / y_{i}=v^{\prime}
$$

Let $v=A_{1}+A_{2} y_{1}$ with $A_{1}, A_{2} \in \mathbf{C}\left(z, y_{2}, \ldots, y_{n}\right)$. Then

$$
\frac{1}{y_{1}}=A_{1}^{\prime}-\sum_{2}^{n} c_{i} \frac{1}{y_{i}}+A_{2}^{\prime} y_{1}+A_{2} \frac{f_{1}^{\prime}(z)}{2 y_{1}}
$$

or

$$
A_{2}^{\prime} y_{1}^{2}+\left(A_{1}^{\prime}-\sum c_{i} \frac{1}{y_{i}}\right) y_{1}+\left(A_{2} \frac{f_{1}^{\prime}(z)}{2}-1\right)=0 .
$$

Since $y_{1}^{2}-f_{1}(z)$ is the minimum polynomial of $y_{1}$ over $\mathbf{C}\left(z, y_{2}, \ldots, y_{n}\right)$, we get $A_{1}^{\prime}-\Sigma c_{i} / y_{i}=0$. Therefore, $1 / y_{1}=v^{\prime}, v$ in $\mathbf{C}\left(z, y_{1}, \ldots, y_{n}\right)$. Taking the trace, with respect to $\mathbf{C}\left(z, y_{1}\right)$, of both sides of this equation, we see that we can assume $1 / y_{1}=v^{\prime}$ with $v$ in $\mathbf{C}\left(z, y_{1}\right)$. This would contradict the fact that $1 / y_{1}$ does not have an elementary integral $[4$, p. 36].

(b) Integrals of the form $\int f \exp (g)$. Let $f_{1}, \ldots, f_{n}, g_{1}, \ldots, g_{n}$ be algebraic over $\mathbf{C}(z)$ and assume $g_{i}-g_{j} \notin \mathbf{C}$ for $i \neq j$. The integrals

$$
\int f_{1} \exp \left(g_{1}\right), \ldots, \int f_{n} \exp \left(g_{n}\right)
$$

satisfy some elementary relation if and only if some $\int f_{i} \exp \left(g_{i}\right)$ is elementary. This follows immediately from Theorem 1 and the following theorem of Liouville (Ritt [4, p. 49]): Let $w=f_{1} \exp \left(g_{1}\right)+\cdots+f_{n}{ }^{2} \exp \left(g_{n}\right)$ with $f_{i}$ and $g_{i}$ as above. $w$ has an elementary integral if and only if each $f_{i} \exp \left(g_{i}\right)$ has an elementary integral.

(c) Inverses of integrals. For completeness, we give this application due to Ritt. If a function satisfies an elementary relation, then so does its inverse. Therefore, if the inverse function of an integral satisfies an elementary relation, the integral is elementary. This shows, in particular, that elliptic functions are not elementary.

5. Mordukhai-Boltovski's Theorem: solutions of differential equations satisfying elementary relations. Let $k$ be a differential field with derivations $\Delta=\{\delta\}$ and constant subfield $\mathcal{C}$. Let $L_{0}=k\left(x_{1}, \ldots, x_{n}, a_{1}, \ldots, a_{m}\right)$ be a 
differential extension of $k$, with the same constants, where $\left\{x_{1}, \ldots, x_{n}\right\}$ is a transcendence base for $L_{0}$ over $k$ and each $a_{i}$ is algebraic over $k\left(x_{1}, \ldots, x_{n}\right)$. Now define a new field $K_{0}=k\left(X_{1}, \ldots, X_{n}, A_{1}, \ldots, A_{m}\right)$ which is isomorphic to $L_{0}$ under the map $\varphi_{0}$ induced by sending $X_{i}$ to $x_{i}$ and $A_{i}$ to $a_{i}$. We can define derivations on $K_{0}$ as follows:

(1) For each $\delta$ in $\Delta$, define a derivation $D$ on $K_{0}$ which agrees with $\delta$ on $k$ and such that $D\left(X_{i}\right)=0$ for $1<i<n$.

(2) For each $X_{i}$ define a derivation $D_{i}$ so that $D_{i}=0$ on $k$ and $D_{i}\left(X_{j}\right)=1$ if $i=j$ and 0 otherwise.

Let $\mathcal{D}$ be the set of derivations of the above two types and consider $K_{0}$ as a differential field with derivations $\mathscr{D}$. The field of constants of $K_{0}$ is precisely $\mathcal{C}$. To see this, first note that the field of constants of $k\left(X_{1}, \ldots, X_{n}\right)$ with respect to the derivations $\mathscr{D}$ must be $\mathcal{C}$. Since any constant $c$ of $K_{0}$ is algebraic over $k\left(X_{1}, \ldots, X_{n}\right), c$ must be algebraic over $\mathcal{C}$. Therefore $\varphi_{0}(c)$ must be algebraic over $\mathcal{C}$, and therefore a constant in $L_{0}$. Thus we can conclude $\varphi_{0}(c)$ is in $\mathcal{C}$ and, since $\varphi_{0}$ is an isomorphism which is the identity map on $\mathcal{C}, c$ is in $\mathcal{C}$.

Let $\mathcal{E}=\left\{D_{1}, \ldots, D_{n}\right\}=$ the set of derivations of the second type. In what follows $\left(K_{0}, L_{0}, \varphi_{0}, \mathcal{E}\right)$ will always be of the type described above.

Before proceeding, we wish to modify our notion of elementary relation. $\left(K_{m}, L_{m}, \varphi\right)$ is said to define a good elementary relation for $\left(K_{0}, L_{0}, \varphi, \mathcal{E}\right)$ if $\left(K_{m}, L_{m}, \varphi\right)$ defines an elementary relation for $\left(K_{0}, L_{0}, \varphi, \mathcal{E}\right)$ and $\varphi$ has the additional property that given any $\delta$ in $\Delta, u$ in $K_{m}$, and assuming that $u$, $D_{1}(u), \ldots, D_{n}(u)$ and $D(u)$ are in the valuation ring of $\varphi$, where $D$ corresponds to $\delta$ as in (1) above, then

$$
\delta(\varphi(u))=\sum_{i=1}^{n} \varphi\left(D_{i}(u)\right) \delta\left(\varphi\left(X_{i}\right)\right)+\varphi(D(u)) .
$$

This equation is just the formal analogue of the chain rule.

THEOREM 2. Let $\left(K_{0}, L_{0}, \varphi_{0}, \mathcal{E}\right)$ be as described above. If there exists a good elementary relation for this quadruple, then there is a triple $\left(K_{n}, L_{n}, \varphi\right)$ which defines an elementary relation for $\left(K_{0}, L_{0}, \varphi_{0}, \mathcal{E}\right)$ and an $\eta$ in $K_{n}$ such that $\varphi$ is defined on $\eta$, with respect to some algebraic extension $K$ of $K_{0}$, $\varphi(\eta)=0$ and $\eta$ is an elementary integral with respect to $K_{0}$.

Proof. Let $\left(\tilde{K}_{n}, \tilde{L}_{n}, \tilde{\varphi}\right)$ define a good elementary relation for $\left(K_{0}, L_{0}, \varphi_{0}\right.$, E). If there is an $R$ in $\tilde{K}_{n}, R \neq 0$, algebraic over $K_{0}$ s.t. $\tilde{\varphi}(R)=0$, we are done, since $R$ is then automatically an elementary integral with respect to an algebraic extension of $K_{0}$. Therefore we can assume that there is no such $R$ and thus that $\tilde{\varphi}$ is an isomorphism on any subfield of $K_{n}$ which is algebraic 
over $K_{0}$. Let $\tilde{\eta} \in K_{n}$ be an element satisfying the conclusion of Lemma 1. If $\tilde{\eta}$ is an elementary integral with respect to some algebraic extension of $K_{0}$, we are again done. If $\tilde{\eta}$ is an exponential of an elementary integral and not algebraic over $K_{0}$, we will want to add a new element, corresponding to $\log \tilde{\eta}$, to our tower and define a new place $\varphi$ such that $\varphi(\log \tilde{\eta})=0$.

More precisely, let $K$ be the algebraic extension of $K_{0}$ containing the elements $U_{1}, \ldots, U_{k}, V$ where $D \tilde{\eta} / \tilde{\eta}=\sum_{i=1}^{l} c_{i} D U_{i} / U_{i}+D V$. We want to adjoin a new element $\eta$ to $K$ such that $D \eta=D \tilde{\eta} / \tilde{\eta}$ for all $D$ in $\mathscr{D}$. In order to get such an element, we will use Proposition 1, so first we must show that there is no $\xi$ in $K$ such that $D \xi=D \tilde{\eta} / \tilde{\eta}$. Assume that there is such an element. Then using the fact that $\tilde{\varphi}$ comes from a good elementary relation, we have

$$
\begin{aligned}
\delta(\tilde{\varphi}(\xi)) & =\sum_{i=1}^{n} \tilde{\varphi}\left(D_{i}(\xi)\right) \delta\left(\tilde{\varphi}\left(X_{i}\right)\right)+\tilde{\varphi}(D(\xi)) \\
& =\sum_{i=1}^{n} \tilde{\varphi}\left(\frac{D_{i} \tilde{\eta}}{\tilde{\eta}}\right) \delta\left(\tilde{\varphi}\left(X_{i}\right)\right)+\tilde{\varphi}\left(\frac{D \tilde{\eta}}{\tilde{\eta}}\right) \\
& =\delta(\tilde{\varphi}(\tilde{\eta})) / \tilde{\varphi}(\tilde{\eta})=0 \text { since } \tilde{\varphi}(\tilde{\eta})=1 .
\end{aligned}
$$

Therefore $\tilde{\varphi}(\xi)$ is a constant. By our above remarks, $\xi$ is a constant, since $\tilde{\varphi}$ is an isomorphism on $K$ and maps the constants of $K$ onto the constants of $L_{0}$. Since $D \xi=D \tilde{\eta} / \tilde{\eta}$, we get that $\tilde{\eta}$ is a constant and so is in $K$. This contradicts the fact that $\tilde{\eta}$ was assumed to be transcendental over $K_{0}$. Therefore, using the discussion following Proposition 1, we can adjoin a transcendental element $\eta$ to $K$, make $K(\eta)$ into a differential field by letting $D \eta=\sum_{i=1}^{l} c_{i} D U_{i} / U_{i}+D V$ for all $D$ in $\mathscr{D}$ and still be assured that we have not added new constants. $\tilde{\varphi}$ is an isomorphism on $K_{0}$, and so is an isomorphism on the algebraic extension $K$. We can extend $\tilde{\varphi}$ to a place $\varphi$ on $K(\eta)$ by setting $\varphi(\eta)=0$. I now claim that $(K(\eta), L, \varphi)$ define the desired elementary relation, where $L=\varphi(K)$. Property 1 is immediate. To check property 2 , we need only check that $\varphi$ is defined on $\eta$, but this follows from the fact that $D \eta=D \tilde{\eta} / \tilde{\eta}, \varphi(\eta)=0$ and $\tilde{\varphi}$ is defined on $\tilde{\eta}$. To check property 3 , let $\alpha$ be in $K(\eta)$ such that $\varphi(\alpha)=0$ and $D \alpha=0$ for all $D$ in $\delta$. Since $\varphi(\alpha)=0$, we can write $\alpha=f / g$ where $f=\eta^{n}\left(a_{m} \eta^{m}+\cdots+a_{0}\right)$ and $g=b_{m} \eta^{m}+\cdots+b_{0}$ where $n>1$, the $a_{i}$ 's and $b_{i}$ 's are in $K$ and $a_{0} b_{0} \neq 0$. Since $D \alpha=0, g D f-$ $f D g=0$ for all $D$ in $\mathcal{E}$. The term of lowest degree in this last expression is $\left(a_{0} b_{0} D \eta\right) \eta^{n-1}$, so $a_{0} b_{0} D \eta=0$. Therefore, $D \eta=0$ for all $D$ in $\mathcal{E}$. This contradicts the fact that $D \eta=D \tilde{\eta} / \tilde{\eta}$ and $D \tilde{\eta} \neq 0$ for some $D$ in $\mathcal{E}$. The $R$ that satisfies property 4 is just $\eta$.

COROLlARY. Let $\mathbf{C}(z)$ be the field of rational functions over $\mathbf{C}$ with the usual derivation, ', where $z^{\prime}=1$ and let $y$ be a solution of the differential equation 
$f\left(z, y, y^{\prime}\right)=0$, where $f$ is a polynomial of degree $>1$ in $y$ and $y^{\prime}$ with coefficients in $\mathbf{C}(z)$. If there exists a nonzero elementary function $g$ of two variables such that $g(z, y)=0$, then there exist nonzero algebraic functions of two variables, $V, U_{1}, \ldots, U_{l}$ and complex numbers $c_{1}, \ldots, c_{l}$ such that

$$
V(z, y)+\sum_{i=1}^{l} c_{i} \log U_{i}(z, y)=0 .
$$

Proof. Let $k=\mathbf{C}(z), L_{0}=k\left(y, y^{\prime}\right)$ and $\Delta=\left\{f^{\prime}\right\} . L_{0}$ is closed under the derivation ' (to see that $L_{0}$ contains $y^{\prime \prime}$, just differentiate $f\left(z, y, y^{\prime}\right)=0$ and solve for $y^{\prime \prime}$ in terms of lower order derivatives of $y$ ). We can assume that $y$ is not algebraic over $k$, otherwise we could find a polynomial $V$ with coefficients in $\mathrm{C}$ such that $V(z, y)=0$. Letting $l=0$, we would have the conclusion of the corollary. Now let $K_{0}=k\left(w, w_{1}\right)$ be a field isomorphic to $L_{0}$ under the map $\varphi_{0}: K_{0} \rightarrow L_{0}$ with $\varphi_{0}(w)=y$ and $\varphi_{0}\left(w_{1}\right)=y^{\prime}$, and turn $K_{0}$ into a differential field as in the first paragraph of this section. Now we proceed as in \$4. We can find a region \& in $C$ such that $y$ and all its derivatives are analytic in $B$. Let $\Re$ be the collection of points $(z, y(z))$ in $\mathbf{C}^{2}$ with $z$ in (S). We can find a region ${ }^{\prime \prime}{ }^{\prime}$ in $\mathbf{C}^{2}$ and an elementary tower $K_{n} \supset \cdots \supset K_{0}$ such that (1) $K_{i}=K_{i-1}\left(\eta_{i}\right)$, where $\eta_{i}$ is analytic in (s' and is either a logarithm, exponential or algebraic over $K_{i-1}$, (2) there is an $F$, not identically zero, in $K_{n}$ such that $F$ is zero on $\Re \cap \cap \mathbb{S}^{\prime}$. We can further assume that $\left\{K_{i}\right\}$ is a minimal tower having properties (1) and (2). Let $\theta=\{G \in$ $K_{n} \mid G$ is defined and finite at some point of $\left.\Re \cap \mathcal{S}^{\prime}\right\}$. This is a valuation ring as before. Define $\varphi$ on $\theta$ as $\varphi(G(z, w))=G(z, y(z))$. This maps $\theta$ onto a field of meromorphic functions on ${ }^{\prime \prime} \subset \subset$ C. The tower $\left\{L_{i}\right\}$ is defined as the image of $\left\{K_{n}\right\} .\left(K_{n}, L_{n}, \varphi\right)$ then defines an elementary relation for $\left(K_{0}, L_{0}, \varphi_{0}\right.$, $\{\partial / \partial w\})$. Since $\varphi$ just substitutes $y$ for $w$, we see that $\left(K_{n}, L_{n}, \varphi\right)$ defines a good elementary relation. Now applying Theorem 2 , we reach the desired conclusion. This corollary was originally proven by Mordukhai-Boltovski [4].

6. Final comments. (I) One would like to generalize Theorem 1 to include exponentials of integrals in the same way that the Kolchin-Ostrowski Theorem does. In particular, if $F$ is a differential field with derivations $\{D\}$ and $\zeta_{1}, \ldots, \zeta_{m}$ are elements in some differential extension such that $D \zeta_{i} / \zeta_{i} \in F$ for each $D$ and such that $\zeta_{1}, \ldots, \zeta_{m}$ satisfy an elementary relation, do there exist integers $v_{1}, \ldots, v_{m}$, not all zero, such that $\Pi_{i=1}^{m} \zeta_{i}^{v_{1}}$ is elementary over $F$ ? The answer is no. Let $w=\int \exp \left(-x^{2}\right)$. It is known [6], that this is not an elementary integral over $\mathbf{C}\left(x, \exp \left(x^{2}\right)\right)$. Let $\zeta_{1}=\exp w, \zeta_{2}=\exp (\pi w), \zeta_{1}$ and $\zeta_{2}$ satisfy the elementary relation $\zeta_{2}-\exp \left(\pi \log \zeta_{1}\right)=0$. If $\zeta_{1}^{\nu_{1}} \zeta_{2}^{\nu_{2}}$ were elementary, then $\left(\nu_{1}+\pi \nu_{2}\right) \int \exp \left(-x^{2}\right)$ would be elementary. Therefore $\nu_{1}=$ $\nu_{2}=0$. 
(II) Theorem 1 says that if an integral satisfies an elementary relation, then it is elementary. This is not true for solutions of first order differential equations. Let $y$ be a function such that $y-\exp \left(y x^{-1}\right)=0$. Liouville showed that this equation does not have Liouvillian solutions (see [7]), much less elementary ones. Yet, we have seen in example (b) of $\$ 2$, that this function satisfies a first order differential equation and an elementary relation. So in some sense Theorem 2 and its corollary are the best one could hope for.

(III) Lemma 1 has been used in [9] to deal with elementary solutions of differential equations. In particular, we showed the following theorem for the case $n=1$ : Let $E$ be a differential extension of a differential field $k$ which is of transcendence degree $n$. If $E$ lies in a generalized elementary extension of $k$, then $E$ lies in a generalized elementary extension of transcendence degree $n$ over $k$. A more direct proof of the full theorem will appear soon.

\section{BIBLIOGRAPHY}

1. J. Ax, On Schanuel's conjectures, Ann. of Math. (2) 93 (1971), 252-268. MR 43 \#3215.

2. C. Chevalley, Introduction to the theory of algebraic functions of one variable, Math. Surveys, no. 6, Amer. Math. Soc., Providence, R. I., 1951. MR 13, 64.

2a. R. Gunning and H. Rossi, Analytic functions of several complex variables, Prentice-Hall, Englewood Cliffs, N. J., 1965. MR 31 \#4927.

3. I. Kaplansky, An introduction to differential algebra, Actualités Sci. Indust., no. $1251=$ Publ. Inst. Math. Univ. Nancago, no. 5, Hermann, Paris, 1957. MR 20 \# 177.

4. J. F. Ritt, Integration in finite terms, Columbia Univ. Press, New York, 1948. MR 9, 573.

5. $\longrightarrow$ On the integrals of elementary functions, Trans. Amer. Math. Soc. 25 (1923), 211-222.

6. M. Rosenlicht, Liouville's theorem on functions with elementary integrals, Pacific J. Math. 24 (1968), 153-161. MR 36 \#6394.

7. On the explicit solvability of certain transcendental equations, Inst. Hautes Ėtudes Sci. Publ. Math. No. 36 (1969), 15-22. MR 41 \# 3454.

8. _ On Liouville's theory of elementary functions, Pacific J. Math. (to appear).

9. M. Singer, Functions satisfying elementary relations, Thesis, Univ. of California, Berkeley, 1974.

Department of Mathematics, State University of New York, Stony Brook, New York 11794

Current address: Department of Mathematics, North Carolina State University, Raleigh, North Carolina 27607 